\title{
Effect of fertilizer levels on nutrient availability and yield of cotton on Vertisol at Rahuri, District Ahemadnagar, India
}

\author{
A. N. Deshpande, R. S. Masram and B. M. Kamble* \\ Department of Soil Science and Agricultural Chemistry, Mahatma Phule Agricultural University, Rahuri, \\ Ahemadnagar- 413722 (Maharashtra), INDIA \\ Present address: Agricultural Research Station, K. Digraj, Sangli- 416305 (Maharashtra), INDIA \\ *Corresponding author: E-mail: bmkamble2007@ rediffmail.com
}

Received: May 23, 2014 Revised received: August 16, 2014 ; Accepted: October 23, 2014

\begin{abstract}
The field experiment on effect of fertilizer levels on nutrient availability and yield of cotton on Vertisol was conducted at Mahatma Phule Krishi Vidyapeeth, Rahuri during May to September, 2008. The experiment was laid out in factorial randomized block design with three replications and two main treatments i.e. hybrid $\mathrm{Bt} \mathrm{RCH}-2$ and Non-Bt $\mathrm{RCH}-2$ and nine sub treatments with different fertilizer doses i.e. $\mathrm{F}_{1}$ - No recommended doses of fertilizer (RDF), $F_{2}-50 \%$ RDF, $F_{3}-75 \%$ RDF, $F_{4}-100 \%$ RDF, $F_{5}-125 \%$ RDF, $F_{6}-150 \%$ RDF, $F_{7}-100 \%$ RDF + $2 \%$ DAP spray, $\mathrm{F}_{8}-100 \% \mathrm{RDF}+1 \% \mathrm{MgSO}_{4}$ and $\mathrm{F}_{9}-100 \% \mathrm{RDF}+1 \% \mathrm{KNO}_{3}$. The results revealed that the non-Bt hybrid recorded maximum nitrogen $\left(435.67 \mathrm{~kg} \mathrm{ha}^{-1}\right)$ and potassium $\left(565.45 \mathrm{~kg} \mathrm{ha}^{-1}\right)$ content in $100 \%$ RDF + $1 \% \mathrm{KNO}_{3}$ treatment at boll development stage as compared to Bt hybrid. However, magnesium $\left(0.09 \mathrm{~kg} \mathrm{ha}^{-1}\right)$ and iron (3.66 ppm) were maximum in $100 \% \mathrm{RDF}+1 \% \mathrm{MgSO}_{4}$ treatment in non-Bt as compared to Bt hybrid and phosphorus content $\left(17.58 \mathrm{~kg} \mathrm{ha}^{-1}\right)$ was maximum in $100 \% \mathrm{RDF}+2 \% \mathrm{DAP}$ treatment in Bt as compared to non-Bt hybrid at boll development stage. The application of $100 \% \mathrm{RDF}+1 \% \mathrm{MgSO}_{4}$ sprays were recorded highest yield of $\mathrm{Bt}$ (33.53 $\left.\mathrm{q} \mathrm{ha}^{-1}\right)$ and non-Bt cotton hybrids $\left(31.27 \mathrm{q} \mathrm{ha}^{-1}\right)$.
\end{abstract}

Keywords: Cotton, Fertilizer, Nutrient availability, Yield

\section{INTRODUCTION}

Cotton is known as white gold and queen of fibers. It is an important cash crop of global significance which plays a dominant role in world agriculture and industrial economy. India is important grower of cotton on a global scale. It is one of the important cash crop for Indian farmer and contributes around 30 per cent to gross domestic product of Indian agriculture. The acreage under cotton in 2012-13, was decreased by around 3\% to 117.73 lakh hectares as against 121.78 lakh hectares in previous year and acreage under Bt cultivation has also declined to $88 \%$ of the total acreage of around 117.73 lakh hectares as against $91 \%$ in last year. The cotton productivity in 2012-13 has been at $518 \mathrm{~kg} \mathrm{ha}^{-1}$ as against $512 \mathrm{~kg} \mathrm{ha}^{-1}$ in the previous season (Anonymous, 2013). Cotton plays an important role in the Indian economy as the country's textile industry is predominantly cotton based. India is one of the largest producers as well as exporters of cotton yarn and the Indian textile industry contributes about 11 percent to industrial production, 14 per cent to the manufacturing sector, 4 percent to the GDP and 12 per cent to the country's total export earnings (Anonymous, 2014). Cotton is one of the important industrial crop playing a vital role in the history of mankind and civilization by providing the basic fibres of typical nature for clothing cellulose from it's lint and oil. Considering this importance of cotton crop different attempts have been made to boost up it's production. To grow the Bt cotton is one of the major attempt which is now a days following everywhere in India, as well as specially in Vidharbha.The commercial cultivation of Bt cotton in the world first began in 1996. Development of transgenic Bt cotton hybrids were approved in India for commercial cultivation in March 2002. Bt cotton has been developed by transferring crystal protein gene (Cry 1AC) from a soil bacterium Bacillus thurigensis var. Kurstaki into cotton. The hybrids with Cry $1 \mathrm{AC}$ gene are known as Boll guard-I which is resistance to bollworms and the hybrids with Cry 1AC gene and Cry 2Ab gene called Boll guard-II. Twenty Bt hybrids have been approved for commercial cultivation in different agro-climatic areas of the country. These hybrids are fast becoming popular with the cotton farmers (Singh and Kaushik, 2007). Though the area under the Bt cotton is substantial but productivity is not of its full potential and considerable reduction in yield of cotton is due to several reasons of abiotic and biotic stresses. The present investigation was undertaken to study the effect of fertilizer levels on 
nutrient availability and yield of cotton.

\section{MATERIALS AND METHODS}

The field experiment was conducted at Cotton Improvement Project Farm, Mahatma Phule Krishi Vidyapeeth, Rahuri during May to September, 2008. The soil of experiment plot was montomorillonitic Typic Haplusterts, deep black with slightly alkaline in reaction $\mathrm{pH}(1: 2.5) 7.8$, electrical conductivity $\left(\mathrm{dS} \mathrm{m}^{-1}\right)$ 0.36 , organic carbon (\%) $0.58, \mathrm{CaCO}_{3}(\%)$ 11.20, available nutrients $\left(\mathrm{kg} \mathrm{ha}^{-1}\right) \mathrm{N}-244.50, \mathrm{P}-12.90$, $\mathrm{K}-526.40$ and $\mathrm{Fe}-4.9, \mathrm{Mn}-8.0, \mathrm{Zn}-0.4, \mathrm{Cu}-2.20$ (ppm). Seeds of Bt and non Bt ( $\mathrm{RCH}-2)$ variety were obtained from private Mahyco seed company. The experiment was laid out in factorial randomized block design with three replications and two main treatments i.e. hybrid $\mathrm{Bt} \mathrm{RCH}-2$ and Non-Bt $\mathrm{RCH}-2$ and nine sub treatments with different fertilizer doses i.e. $\mathrm{F}_{1}$ No RDF, $\mathrm{F}_{2}-50 \%$ RDF, $\mathrm{F}_{3}-75 \%$ RDF, $\mathrm{F}_{4}-100 \%$ $\mathrm{RDF}, \mathrm{F}_{5}-125 \% \mathrm{RDF}, \mathrm{F}_{6}-150 \% \mathrm{RDF}, \mathrm{F}_{7}-100 \%$ $\mathrm{RDF}+2 \%$ DAP spray, $\mathrm{F}_{8}-100 \% \mathrm{RDF}+1 \% \mathrm{MgSO}_{4}$ and $\mathrm{F}_{9}-100 \% \mathrm{RDF}+1 \% \mathrm{KNO}_{3}$. The common RDF for $\mathrm{Bt}$ as well as non $\mathrm{Bt}$ was $100 \mathrm{~N}, 50 \mathrm{P}_{2} \mathrm{O}_{5}$ and 50 $\mathrm{K}_{2} \mathrm{O} \mathrm{kg} \mathrm{ha}{ }^{-1}$. Uniform $10 \mathrm{Mg} \mathrm{ha}^{-1} \mathrm{FYM}$ dose was applied to all 9 treatments including control. Total 3 sprays were given in treatment $F_{7}, F_{8}$ and $F_{9}, 1^{\text {st }}$ at boll development stage and further two sprays at 15 days interval thereafter. The common packages of practices were followed for both cotton hybrids. The nitrogen, phosphorus and potassium were applied through urea, single super phosphate and muriate of potash respectively as per recommended dose 50 per cent of nitrogen and full dose of $\mathrm{P}_{2} \mathrm{O}_{5}$ and $\mathrm{K}_{2} \mathrm{O}$ (50:50:50 $\mathrm{N}: \mathrm{P}_{2} \mathrm{O}_{5}: \mathrm{K}_{2} \mathrm{O} \mathrm{kg} \mathrm{ha-1}$ ) was applied at the time of sowing and the remaining nitrogen was applied in two doses. The composite soil sample before sowing was taken and the soil samples were collected from each plot at square formation, boll formation and boll development stages and dried in shade and prepared for laboratory analysis after grinding and passing through $2 \mathrm{~mm}$ sieve. The seed cotton from each net plot was picked and the same weighed separately at each picking. The total yield $\left(\mathrm{q} \mathrm{ha} \mathrm{h}^{-1}\right.$ ) was work out by summation of quantity of seed cotton picked in all pickings. Plot wise data on soil nutrient content and yield etc. was subjected to statistical analysis by statistical method for factorial randomized block design and accordingly interpretations of results were given (Panse and Sukhatme, 1985).

\section{RESULTS AND DISCUSSION}

Effect of fertilizer levels and nutrient sprays on soil parameters at different growth stages :

Available nitrogen: The nitrogen content in the soil progressively decreased from square formation to boll formation stage (Table 1). Non Bt hybrid recorded maximum content of soil nitrogen (236.54 kg ha-1), $\left(230.46 \mathrm{~kg} \mathrm{ha}^{-1}\right)$ and $\left(312.37 \mathrm{~kg} \mathrm{ha}^{-1}\right)$ as compared to Bt hybrid (230.36 kg ha $\left.{ }^{-1}\right),\left(226.1 \mathrm{~kg} \mathrm{ha}^{-1}\right)$ and (294.86 $\mathrm{kg} \mathrm{ha}{ }^{-1}$ ) from square formation to boll development stages. The treatment $\mathrm{F}_{9}$ recorded maximum nitrogen content in the soil at square formation $\left(245.87 \mathrm{~kg} \mathrm{ha}^{-1}\right)$, boll formation (239.87 $\mathrm{kg} \mathrm{ha}^{-1}$ ) and at boll development stage (428.90 kg ha $\left.{ }^{-1}\right)$ and this was significantly superior over all treatments at square formation and at par with $\mathrm{F}_{8}\left(238.89 \mathrm{~kg} \mathrm{ha}^{-1}\right)$ at boll formation and with $\mathrm{F}_{7}$ (428.38 $\left.\mathrm{kg} \mathrm{ha}^{-1}\right)$ at boll development stage. Nitrogen supply through foliar spray also stated that nitrogen

Table 1. Effect of levels of N, P, K and foliar sprays of nutrients on soil available nitrogen of Bt and non- Bt Cotton.

\begin{tabular}{|c|c|c|c|c|c|c|c|c|c|}
\hline \multirow[t]{2}{*}{ Treatment } & \multicolumn{3}{|c|}{ Square formation } & \multicolumn{3}{|c|}{ Boll formation } & \multicolumn{3}{|c|}{ Boll development } \\
\hline & $\mathbf{H}_{1:} \mathbf{B t}$ & $\begin{array}{c}\mathrm{H}_{2}: \\
\text { Non-Bt }\end{array}$ & Mean & $\mathbf{H}_{1:} \mathbf{B t}$ & $\begin{array}{c}\mathbf{H}_{2}: \\
\text { Non - Bt }\end{array}$ & Mean & $\mathbf{H}_{1:} \mathbf{B t}$ & $\begin{array}{c}\mathbf{H}_{2}: \\
\text { Non-Bt }\end{array}$ & Mean \\
\hline & \multicolumn{9}{|c|}{ Available nitrogen $\left(\mathrm{kg} \mathrm{ha}^{-1}\right)$} \\
\hline $\mathrm{F}_{1}$ : Control & 199.80 & 114.73 & 207.26 & 196.30 & 200.01 & 198.15 & 160.00 & 193.06 & 177.53 \\
\hline $\mathrm{F}_{2}: 50 \% \mathrm{RDF}$ & 219.33 & 227.88 & 223.60 & 217.99 & 224.25 & 221.10 & 295.97 & 208.03 & 202.00 \\
\hline $\mathrm{F}_{3}: 75 \% \mathrm{RDF}$ & 225.37 & 235.63 & 230.50 & 221.65 & 226.12 & 233.88 & 298.67 & 218.60 & 208.7 \\
\hline $\mathrm{F}_{4}: 100 \% \mathrm{RDF}$ & 239.70 & 239.30 & 239.50 & 234.03 & 237.87 & 235.95 & 410.97 & 429.37 & 420.17 \\
\hline $\mathrm{F}_{5}: 125 \% \mathrm{RDF}$ & 227.26 & 239.03 & 233.14 & 225.83 & 231.77 & 228.79 & 207.58 & 227.67 & 217.62 \\
\hline $\mathrm{F}_{6}: 150 \% \mathrm{RDF}$ & 235.10 & 241.68 & 238.39 & 228.92 & 235.45 & 232.19 & 218.71 & 231.37 & 225.04 \\
\hline $\begin{array}{l}\mathrm{F}_{7}: \mathrm{RDF}+2 \% \\
\text { DAP spray }\end{array}$ & 240.97 & 242.90 & 241.43 & 235.21 & 240.47 & 237.84 & 421.47 & 435.30 & 428.38 \\
\hline $\begin{array}{l}\mathrm{F}_{8}: \mathrm{RDF}+1 \% \\
\mathrm{MgSO}_{4} \text { spray }\end{array}$ & 240.67 & 241.09 & 241.36 & 237.18 & 240.50 & 238.89 & 418.26 & 430.27 & 424.27 \\
\hline $\begin{array}{l}\mathrm{F}_{9}: \mathrm{RDF}+1 \% \\
\mathrm{KNO}_{3} \text { spray }\end{array}$ & 245.05 & 246.69 & 245.87 & 238.43 & 241.31 & 239.87 & 242.13 & 435.67 & 428.90 \\
\hline Mean & 230.36 & 236.54 & 233.45 & 226.11 & 230.46 & 228.51 & 294.86 & 312.37 & 303.62 \\
\hline & $\mathrm{H}$ & $\bar{F}$ & $\mathrm{HX} \mathrm{F}$ & $\mathrm{H}$ & $\mathrm{F}$ & $\mathrm{HX} \mathrm{F}$ & $\mathrm{H}$ & $\mathrm{F}$ & $\mathrm{H} \mathrm{X} \mathrm{F}$ \\
\hline $\mathrm{SEM}_{-}^{+}$ & 0.56 & 1.19 & 1.69 & 0.20 & 0.43 & 0.61 & 0.12 & 0.26 & 0.36 \\
\hline $\mathrm{CD}$ at $5 \%$ & 1.62 & 3.44 & 4.86 & 0.58 & 1.24 & 1.75 & 0.35 & 0.75 & 1.05 \\
\hline
\end{tabular}


Table 2. Effect of levels of N, P, K and foliar sprays of nutrients on soil available phosphorus of Bt and non- Bt Cotton.

\begin{tabular}{|c|c|c|c|c|c|c|c|c|c|}
\hline \multirow[t]{2}{*}{ Treatment } & \multicolumn{3}{|c|}{ Square formation } & \multicolumn{3}{|c|}{ Boll formation } & \multicolumn{3}{|c|}{ Boll development } \\
\hline & $\mathbf{H}_{1:} \mathbf{B t}$ & $\begin{array}{c}\mathbf{H}_{2}: \\
\text { Non-Bt }\end{array}$ & Mean & $\mathrm{H}_{1:} \mathbf{B t}$ & $\begin{array}{c}\mathrm{H}_{2}: \\
\text { Non - Bt }\end{array}$ & Mean & $\mathbf{H}_{1:} \mathbf{B t}$ & $\begin{array}{c}\mathrm{H}_{2}: \\
\text { Non-Bt }\end{array}$ & Mean \\
\hline & \multicolumn{9}{|c|}{ Available phosphorus $\left(\mathrm{kg} \mathrm{ha}^{-1}\right)$} \\
\hline $\mathrm{F}_{1}$ : Control & 14.10 & 14.00 & 14.05 & 15.01 & 14.82 & 14.91 & 16.81 & 16.50 & 16.66 \\
\hline $\mathrm{F}_{2}: 50 \% \mathrm{RDF}$ & 14.51 & 14.26 & 14.38 & 15.37 & 15.01 & 15.19 & 17.06 & 16.96 & 17.01 \\
\hline $\mathrm{F}_{3}: 75 \% \mathrm{RDF}$ & 14.57 & 14.46 & 14.51 & 15.48 & 15.26 & 15.37 & 17.17 & 17.05 & 17.11 \\
\hline $\mathrm{F}_{4}: 100 \% \mathrm{RDF}$ & 14.80 & 14.63 & 14.71 & 15.65 & 15.60 & 15.62 & 17.77 & 17.62 & 17.69 \\
\hline $\mathrm{F}_{5}: 125 \% \mathrm{RDF}$ & 14.66 & 14.55 & 14.61 & 15.54 & 15.37 & 15.46 & 17.30 & 17.23 & 17.26 \\
\hline $\mathrm{F}_{6}: 150 \% \mathrm{RDF}$ & 14.77 & 14.61 & 14.69 & 15.60 & 15.43 & 15.52 & 17.58 & 17.43 & 17.50 \\
\hline $\begin{array}{l}\mathrm{F}_{7}: \mathrm{RDF}+2 \% \\
\text { DAP spray }\end{array}$ & 14.87 & 14.72 & 14.79 & 15.66 & 15.61 & 15.63 & 18.10 & 18.09 & 18.09 \\
\hline $\begin{array}{l}\mathrm{F}_{8}: \mathrm{RDF}+1 \% \\
\mathrm{MgSO}_{4} \text { spray }\end{array}$ & 14.82 & 14.65 & 14.73 & 15.66 & 15.62 & 15.64 & 17.89 & 17.69 & 17.79 \\
\hline $\begin{array}{l}\mathrm{F}_{9}: \mathrm{RDF}+1 \% \\
\mathrm{KNO}_{3} \text { spray }\end{array}$ & 14.83 & 14.67 & 14.75 & 15.66 & 15.65 & 15.65 & 18.15 & 18.02 & 18.09 \\
\hline \multirow[t]{2}{*}{ Mean } & 14.66 & 14.50 & 15.68 & 15.52 & 15.37 & 15.44 & 17.54 & 17.40 & 17.47 \\
\hline & $\mathrm{H}$ & $\mathrm{F}$ & H X F & $\mathrm{H}$ & $\mathrm{F}$ & H X F & $\mathrm{H}$ & $\bar{F}$ & H X F \\
\hline SEM - & 0.003 & 0.007 & 0.010 & 0.003 & 0.007 & 0.010 & 0.010 & 0.022 & 0.31 \\
\hline $\mathrm{CD}$ at $5 \%$ & 0.010 & 0.021 & 0.029 & 0.009 & 0.020 & 0.028 & 0.030 & 0.063 & 0.089 \\
\hline
\end{tabular}

Table 3. Effect of levels of N,P.K and foliar sprays of nutrients on soil available potassium of Bt and non- Bt cotton

\begin{tabular}{|c|c|c|c|c|c|c|c|c|c|}
\hline \multirow[t]{2}{*}{ Treatment } & \multicolumn{3}{|c|}{ Square formation } & \multicolumn{3}{|c|}{ Boll formation } & \multicolumn{3}{|c|}{ Boll development } \\
\hline & $\mathbf{H}_{1:} \mathbf{B t}$ & $\begin{array}{c}\mathbf{H}_{2}: \\
\text { Non-Bt }\end{array}$ & Mean & $\mathbf{H}_{1:} \mathbf{B t}$ & $\begin{array}{c}\mathbf{H}_{2}: \\
\text { Non-Bt }\end{array}$ & Mean & $\mathbf{H}_{1:} \mathbf{B t}$ & $\begin{array}{c}\mathbf{H}_{2}: \\
\text { Non-Bt }\end{array}$ & Mean \\
\hline & \multicolumn{9}{|c|}{ Available potassium $\left(\mathrm{kg} \mathrm{ha}^{-1}\right)$} \\
\hline $\mathrm{F}_{1}$ : Control & 515.07 & 521.80 & 518.43 & 490.09 & 505.70 & 497.89 & 440.90 & 496.37 & 468.63 \\
\hline $\mathrm{F}_{2}: 50 \% \mathrm{RDF}$ & 526.93 & 544.30 & 535.62 & 552.14 & 526.21 & 599.17 & 482.27 & 508.43 & 496.85 \\
\hline $\mathrm{F}_{3}: 75 \% \mathrm{RDF}$ & 538.33 & 550.08 & 544.21 & 528.27 & 531.00 & 529.63 & 503.40 & 515.14 & 509.27 \\
\hline $\mathrm{F}_{4}: 100 \% \mathrm{RDF}$ & 559.73 & 562.03 & 560.88 & 550.49 & 5.54 .27 & 552.38 & 534.33 & 542.14 & 538.24 \\
\hline $\mathrm{F}_{5}: 125 \% \mathrm{RDF}$ & 545.28 & 556.37 & 550.82 & 535.77 & 537.78 & 537.78 & 515.53 & 524.33 & 520.43 \\
\hline $\mathrm{F}_{6}: 150 \% \mathrm{RDF}$ & 558.27 & 560.70 & 559.48 & 548.33 & 548.24 & 548.28 & 528.00 & 535.33 & 531.66 \\
\hline $\begin{array}{l}\mathrm{F}_{7}: \mathrm{RDF}+2 \% \\
\text { DAP spray }\end{array}$ & 561.33 & 564.05 & 562.69 & 558.24 & 557.90 & 558.07 & 550.00 & 552.16 & 551.08 \\
\hline $\begin{array}{l}\mathrm{F}_{8}: \mathrm{RDF}+1 \% \\
\mathrm{MgSO}_{4} \text { spray }\end{array}$ & 560.10 & 565.23 & 562.67 & 545.77 & 553.77 & 554.21 & 542.27 & 550.54 & 546.40 \\
\hline $\begin{array}{l}\mathrm{F}_{9}: \mathrm{RDF}+1 \% \\
\mathrm{KNO}_{3} \text { spray }\end{array}$ & 566.20 & 567.77 & 566.98 & 558.27 & 561.63 & 559.95 & 561.93 & 565.45 & 563.69 \\
\hline \multirow[t]{2}{*}{ Mean } & 547.92 & 554.70 & 551.31 & 537.37 & 541.83 & 539.60 & 517.96 & 532.32 & 525.14 \\
\hline & $\mathrm{H}$ & $\mathrm{F}$ & HX F & $\mathrm{H}$ & $\bar{F}$ & HX F & $\mathrm{H}$ & $\mathrm{F}$ & HX F \\
\hline SEM - & 0.19 & 0.39 & 0.56 & 0.23 & 0.49 & 0.69 & 0.21 & 0.45 & 0.64 \\
\hline $\mathrm{CD}$ at $5 \%$ & 0.54 & 1.14 & 1.61 & 0.66 & 1.41 & 1.99 & 0.61 & 1.31 & 1.84 \\
\hline
\end{tabular}

acts as promoter of vegetative growth of leaf, stem and other vegetative organs (Jadhao et al., 2004). Ravankar et al. (2001) also observed that lower doses of nutrients decreased the yields as well as nutrient uptake by the crops. The interaction between $\mathrm{Bt} /$ non $\mathrm{Bt}$ with different fertilizer dose was found significant at all growth stages. Treatment combination of $\mathrm{H}_{2} \mathrm{~F}_{9}$ recorded maximum soil nitrogen content at square formation (246.69 $\left.\mathrm{kg} \mathrm{ha}^{-1}\right)$, boll formation (241.31 $\mathrm{kg} \mathrm{ha}^{-1}$ ) and boll development $\left(435.67 \mathrm{kgha}^{-1}\right)$ stages, however it was at par with $\mathrm{H}_{2} \mathrm{~F}_{7}\left(242.90 \mathrm{~kg} \mathrm{ha}^{-1}\right)$ at square formation $\mathrm{H}_{2} \mathrm{~F}_{8}(240.50$ $\left.\mathrm{kg} \mathrm{ha}^{-1}\right)$ and $\mathrm{H}_{2} \mathrm{~F}_{7}\left(240.47 \mathrm{~kg} \mathrm{ha}^{-1}\right)$ at boll formation. This clearly indicated that, the residual effect of soil nitrogen was more in non-Bt cotton hybrids than $\mathrm{Bt}$ cotton hybrids.

Available phosphorus: The phosphorus content in the 
Table 4. Effect of levels of N,P,K and foliar sprays of nutrients on soil exchangeable magnesium of Bt and non- Bt cotton hybrid cotton.

\begin{tabular}{|c|c|c|c|c|c|c|c|c|c|}
\hline \multirow[t]{2}{*}{ Treatment } & \multicolumn{3}{|c|}{ Square formation } & \multicolumn{3}{|c|}{ Boll formation } & \multicolumn{3}{|c|}{ Boll development } \\
\hline & $\mathrm{H}_{1:} \mathbf{B t}$ & $\begin{array}{c}\mathbf{H}_{2}: \\
\text { Non- Bt }\end{array}$ & Mean & $\mathbf{H}_{1:} \mathbf{B t}$ & $\begin{array}{c}\mathrm{H}_{2}: \\
\text { Non -Bt }\end{array}$ & Mean & $\mathbf{H}_{1:} \mathbf{B t}$ & $\begin{array}{c}\mathrm{H}_{2}: \\
\text { Non -Bt }\end{array}$ & Mean \\
\hline & \multicolumn{9}{|c|}{ Exchangeable magnesium $\left(\mathrm{kg} \mathrm{ha}^{-1}\right)$} \\
\hline $\mathrm{F}_{1}$ : Control & 1.03 & 1.11 & 1.07 & 0.01 & 0.007 & 0.04 & 0.01 & 0.07 & 0.04 \\
\hline $\mathrm{F}_{2}: 50 \% \mathrm{RDF}$ & 1.11 & 1.15 & 1.13 & 0.05 & 0.12 & 0.09 & 0.04 & 0.08 & 0.06 \\
\hline $\mathrm{F}_{3}: 75 \% \mathrm{RDF}$ & 1.14 & 1.19 & 1.16 & 0.09 & 0.12 & 0.11 & 0.06 & 0.08 & 0.07 \\
\hline $\mathrm{F}_{4}: 100 \% \mathrm{RDF}$ & 1.24 & 1.25 & 1.24 & 0.15 & 0.16 & 0.16 & 0.07 & 0.09 & 0.08 \\
\hline $\mathrm{F}_{5}: 125 \% \mathrm{RDF}$ & 1.16 & 1.21 & 1.18 & 0.11 & 0.13 & 0.12 & 0.06 & 0.09 & 0.07 \\
\hline $\mathrm{F}_{6}: 150 \% \mathrm{RDF}$ & 1.21 & 1.24 & 1.23 & 0.13 & 0.14 & 0.13 & 0.07 & 0.09 & 0.08 \\
\hline $\begin{array}{l}\mathrm{F}_{7}: \mathrm{RDF}+2 \% \\
\mathrm{DAP} \text { spray }\end{array}$ & 1.28 & 1.30 & 1.29 & 0.16 & 0.18 & 0.17 & 0.07 & 0.09 & 0.08 \\
\hline $\begin{array}{l}\mathrm{F}_{8}: \mathrm{RDF}+1 \% \\
\mathrm{MgSO}_{4} \text { spray }\end{array}$ & 1.30 & 1.31 & 1.30 & 0.17 & 0.19 & 0.18 & 0.08 & 0.09 & 0.09 \\
\hline $\begin{array}{l}\mathrm{F}_{9}: \mathrm{RDF}+1 \% \\
\mathrm{KNO}_{3} \text { spray }\end{array}$ & 1.28 & 1.29 & 1.28 & 0.17 & 0.18 & 0.18 & 0.07 & 0.09 & 0.08 \\
\hline Mean & 1.19 & 1.23 & 1.21 & 0.12 & 0.14 & 0.13 & 0.06 & 0.08 & 0.07 \\
\hline & $\mathrm{H}$ & $\mathrm{F}$ & H X F & $\mathrm{H}$ & $\mathrm{F}$ & H X F & $\mathrm{H}$ & $\mathrm{F}$ & $\mathrm{H} \mathrm{X} \mathrm{F}$ \\
\hline $\mathrm{SEM}_{-}^{+}$ & 0.004 & 0.008 & 0.011 & 0.002 & 0.004 & 0.005 & 0.000 & 0.001 & 0.001 \\
\hline $\mathrm{CD}$ at $5 \%$ & 0.01 & 0.02 & 0.03 & 0.005 & 0.010 & 0.014 & 0.001 & 0.002 & 0.002 \\
\hline
\end{tabular}

Table 5. Effect of levels of N,P.K and foliar sprays of nutrients on DTPA extractable iron of Bt and non- Bt cotton.

\begin{tabular}{|c|c|c|c|c|c|c|c|c|c|}
\hline \multirow[t]{2}{*}{ Treatment } & \multicolumn{3}{|c|}{ Square formation } & \multicolumn{3}{|c|}{ Boll formation } & \multicolumn{3}{|c|}{ Boll development } \\
\hline & $\mathrm{H}_{1:} \mathbf{B t}$ & $\begin{array}{c}\mathbf{H}_{2}: \\
\text { Non-Bt }\end{array}$ & Mean & $\mathbf{H}_{1:} \mathbf{B t}$ & $\begin{array}{c}\mathbf{H}_{2}: \\
\text { Non-Bt }\end{array}$ & Mean & $\mathbf{H}_{1:} \mathbf{B t}$ & $\begin{array}{c}\mathrm{H}_{2}: \\
\text { Non-Bt }\end{array}$ & Mean \\
\hline & \multicolumn{9}{|c|}{ DTPA Iron (ppm) } \\
\hline $\mathrm{F}_{1}$ : Control & 4.00 & 4.06 & 4.03 & 3.38 & 3.51 & 3.45 & 2.52 & 3.00 & 2.76 \\
\hline $\mathrm{F}_{2}: 50 \% \mathrm{RDF}$ & 4.29 & 4.61 & 4.30 & 3.68 & 4.20 & 3.94 & 3.01 & 3.24 & 3.12 \\
\hline $\mathrm{F}_{3}: 75 \% \mathrm{RDF}$ & 4.39 & 4.47 & 4.43 & 3.73 & 4.25 & 3.99 & 3.08 & 3.28 & 3.18 \\
\hline $\mathrm{F}_{4}: 100 \% \mathrm{RDF}$ & 4.59 & 4.61 & 4.60 & 4.52 & 4.62 & 4.57 & 3.32 & 3.46 & 3.39 \\
\hline $\mathrm{F}_{5}: 125 \% \mathrm{RDF}$ & 4.46 & 4.49 & 4.47 & 4.36 & 4.38 & 4.37 & 3.16 & 3.39 & 3.27 \\
\hline $\mathrm{F}_{6}: 150 \% \mathrm{RDF}$ & 4.54 & 4.61 & 4.57 & 4.44 & 4.43 & 4.44 & 3.24 & 3.41 & 3.32 \\
\hline $\begin{array}{l}\mathrm{F}_{7}: \mathrm{RDF}+2 \% \\
\text { DAP spray }\end{array}$ & 4.80 & 4.84 & 4.82 & 4.76 & 4.80 & 4.78 & 3.43 & 3.59 & 3.51 \\
\hline $\begin{array}{l}\mathrm{F}_{8}: \mathrm{RDF}+1 \% \\
\mathrm{MgSO}_{4} \text { spray }\end{array}$ & 4.70 & 4.71 & 4.71 & 4.72 & 4.71 & 4.71 & 3.46 & 3.66 & 3.56 \\
\hline $\begin{array}{l}\mathrm{F}_{9}: \mathrm{RDF}+1 \% \\
\mathrm{KNO}_{3} \text { spray }\end{array}$ & 4.75 & 4.76 & 4.75 & 4.60 & 4.70 & 4.65 & 3.39 & 3.57 & 3.48 \\
\hline \multirow[t]{2}{*}{ Mean } & 4.50 & 4.54 & 4.52 & 4.24 & 4.40 & 4.32 & 3.18 & 3.40 & 3.29 \\
\hline & $\mathrm{H}$ & $\mathrm{F}$ & H X F & $\mathrm{H}$ & $\mathrm{F}$ & H X F & $\mathrm{H}$ & $\mathrm{F}$ & HX F \\
\hline SEM _- & 0.003 & 0.007 & 0.009 & 0.006 & 0.013 & 0.018 & 0.004 & 0.009 & 0.012 \\
\hline $\mathrm{CD}$ at $5 \%$ & 0.009 & 0.019 & 0.027 & 0.017 & 0.036 & 0.051 & 0.012 & 0.025 & 0.035 \\
\hline
\end{tabular}

soil was slightly increased from square formation to boll development stage (Table 2). Bt hybrid was recorded maximum content of phosphorus $(14.66 \mathrm{~kg}$ $\left.\mathrm{ha}^{-1}\right),\left(15.52 \mathrm{~kg} \mathrm{ha}^{-1}\right)$ and $\left(17.54 \mathrm{~kg} \mathrm{ha}^{-1}\right)$ as compared to non Bt hybrid (14.50 $\left.\mathrm{kg} \mathrm{ha}^{-1}\right),\left(15.37 \mathrm{~kg} \mathrm{ha}^{-1}\right)$ and $\left(17.40 \mathrm{~kg} \mathrm{ha}^{-1}\right)$ at square formation, boll formation and development stage, respectively. The treatment $\mathrm{F}_{7}$ recorded maximum content of phosphorus (14.79 $\mathrm{kg} \mathrm{ha}^{-1}$ ), however, there was not so much difference among the other treatments except $F_{1}\left(14.05 \mathrm{~kg} \mathrm{ha}^{-1}\right)$, $F_{2}\left(14.38 \mathrm{~kg} \mathrm{ha}^{-1}\right)$ and $F_{3}\left(14.51 \mathrm{~kg} \mathrm{ha}^{-1}\right)$ at square formation. Similar observations were recorded at boll formation and boll development stage. Phosphorus availability in soil increased with each 
Table 6. Effect of levels of N, P, K and foliar sprays of nutrients on seed cotton yield of Bt and non- Bt cotton.

\begin{tabular}{|c|c|c|c|}
\hline Treatment & $\mathrm{H}_{1:} \mathbf{B t}$ & $\mathrm{H}_{2}:$ Non $-\mathrm{Bt}$ & Mean \\
\hline & \multicolumn{3}{|c|}{ Yield $\left(q\right.$ ha $\left.^{-1}\right)$} \\
\hline $\mathrm{F}_{1}$ : Control & 15.89 & 14.40 & 15.14 \\
\hline $\mathrm{F}_{2}: 50 \% \mathrm{RDF}$ & 27.82 & 25.25 & 26.54 \\
\hline $\mathrm{F}_{3}: 75 \% \mathrm{RDF}$ & 29.11 & 27.16 & 28.16 \\
\hline $\mathrm{F}_{4}: 100 \% \mathrm{RDF}$ & 30.86 & 28.39 & 29.62 \\
\hline $\mathrm{F}_{5}: 125 \% \mathrm{RDF}$ & 29.93 & 27.72 & 28.83 \\
\hline $\mathrm{F}_{6}: 150 \% \mathrm{RDF}$ & 30.45 & 27.82 & 29.14 \\
\hline $\mathrm{F}_{7}: \mathrm{RDF}+2 \%$ DAP spray & 31.68 & 29.16 & 30.42 \\
\hline $\mathrm{F}_{8}: \mathrm{RDF}+1 \% \mathrm{MgSO}_{4}$ spray & 33.53 & 31.27 & 32.40 \\
\hline $\mathrm{F}_{9}: \mathrm{RDF}+1 \% \mathrm{KNO}_{3}$ spray & 32.35 & 29.98 & 31.17 \\
\hline \multirow[t]{2}{*}{ Mean } & 29.07 & 26.80 & 27.83 \\
\hline & $\mathrm{H}$ & $\mathrm{F}$ & H X F \\
\hline $\mathrm{SEM}_{-}^{+}$ & 0.56 & 1.23 & 1.69 \\
\hline $\mathrm{CD}$ at $5 \%$ & 1.68 & 3.69 & N.S. \\
\hline
\end{tabular}

increment of fertilizer dose. However, increase in availability was not proportionate to added amount .Phosphorus availability in soil also increased with advancement in crop age. This could be ascribed to increase in root activity in soil. Plant roots excrete organic acids and chelating organic compounds in rhizosphere. These compounds form multiple complex compounds with $\mathrm{Ca}, \mathrm{Mg}$ and/or $\mathrm{Fe}$ and thereby increased phosphorus availability in soil (Tinker, 1980). As regards the interaction effect between $\mathrm{Bt} / \mathrm{non} \mathrm{Bt}$ with different fertilizer levels was found to be significant at all growth stages. Treatment combination $\mathrm{H}_{1} \mathrm{~F}_{7}$ (14.87) recorded maximum soil available phosphorus content as compared to the other treatment combinations at square formation. More or less same trend was observed at boll formation and boll development stage. This clearly indicated that, more soil available phosphorus residue was under $\mathrm{Bt}$ cotton hybrid than non $\mathrm{Bt}$ cotton hybrid, which showed the greater absorption of soil phosphorus by non $\mathrm{Bt}$ than $\mathrm{Bt}$ as reflected in phosphorus leaf content of $\mathrm{Bt}$ and non $\mathrm{Bt}$ cotton. Jayakumar et al. (2014) showed the highest NPK of post harvest soil values were noticed with 150 per cent recommended dose of NPK and biofertigation. Higher availability of nutrients in the soil and thereby increased the nutrient uptake by the crop .

Available potassium: The available potassium content in the soil was decreased from square formation to boll development stage. Non Bt hybrid recorded maximum content of soil available potassium $\left(554.70 \mathrm{~kg} \mathrm{ha}^{-1}\right)$, $\left(541.83 \mathrm{~kg} \mathrm{ha}^{-1}\right)$ and $\left(532.32 \mathrm{~kg} \mathrm{ha}^{-1}\right)$ as compared to Bt hybrid (547.92 kg ha-1), (537.37 kg ha-1) and $\left(517.96 \mathrm{~kg} \mathrm{ha}^{-1}\right)$ at square formation, boll formation and boll development stage, respectively (Table 3). The soil available potassium content was more under treatment $\mathrm{F}_{9}$ at square and boll formation stages $\left(566.98\right.$ and $\left.559.95 \mathrm{~kg} \mathrm{ha}^{-1}\right)$ as well as at boll development stage $\left(563.69 \mathrm{~kg} \mathrm{ha}^{-1}\right)$. Similarly, Islamov and Ishmukhame (1967) observed that reddening due to potassium deficiency can be reduced 50 per cent if $200 \mathrm{~kg} \mathrm{~K} \mathrm{ha}{ }^{-1}$ was applied to soil before planting. As regards, the interaction between $\mathrm{Bt} /$ non $\mathrm{Bt}$ with different fertilizer levels was found to be significant at all growth stages. Treatment combination $\mathrm{H}_{2} \mathrm{~F}_{9}(567.77$ $\mathrm{kg} \mathrm{ha}^{-1}$ ) recorded maximum soil available potassium content as compared to $\mathrm{H}_{1} \mathrm{~F}_{9}\left(566.20 \mathrm{~kg} \mathrm{ha}^{-1}\right)$ and it was statistically superior over all other treatment combinations at square formation. Similar observations also recorded at boll formation and boll development stage $\mathrm{H}_{2} \mathrm{~F}_{9}\left(561.63\right.$ and $565.45 \mathrm{~kg} \mathrm{ha}^{-1}$ ) was significantly superior over all other treatments tested. Soil application of potassium as well as foliar spray of fertilizers containing both these nutrients helps to maintain sufficient level of both $\mathrm{N}$ and $\mathrm{K}$ during boll development (Sekhon and Singh, 2013).

Exchangeable magnesium: The magnesium content in the soil was decreased from square formation to boll development stages. Non $\mathrm{Bt}$ hybrid was recorded maximum content of magnesium in the soil (1.23 $\left.\mathrm{kg} \mathrm{ha}^{-1}\right),\left(0.14 \mathrm{~kg} \mathrm{ha}^{-1}\right)$ and $\left(0.08 \mathrm{~kg} \mathrm{ha}^{-1}\right)$ as compared to Bt cotton $\left(1.19 \mathrm{~kg} \mathrm{ha}^{-1}\right),\left(0.12 \mathrm{~kg} \mathrm{ha}^{-1}\right)$ and $\left(0.06 \mathrm{~kg} \mathrm{ha}^{-1}\right)$ at square formation, boll formation and boll development stage, respectively (Table 4). The treatment $\mathrm{F}_{8}$ recorded maximum magnesium content in the soil $\left(1.30 \mathrm{~kg} \mathrm{ha}^{-1}\right)$ and it remained at par with $\mathrm{F}_{7}$ and $\mathrm{F}_{9}$ and treatment $\mathrm{F}_{8}$ was statistically superior over the control $\mathrm{F}_{1}\left(1.07 \quad \mathrm{~kg}^{\mathrm{h}} \mathrm{ha}^{-1}\right)$ at square formation. Concentration of $\mathrm{Mg}$ strongly responded $\mathrm{N}$ fertilization suggesting that cotton $\mathrm{Mg}$ nutrition depends on nitrogen fertilization more than the level of the $\mathrm{Mg}$ application (Tewolde et al., 2010). Magnesium content in the soil was decreased as the growth stages increased. Similar observation recorded at boll formation stage. The $\mathrm{H}_{2} \mathrm{~F}_{8}$ was recorded maximum magnesium content in the soil at square formation $\left(1.31 \mathrm{~kg} \mathrm{ha}^{-1}\right)$, boll formation $\left(0.19 \mathrm{~kg} \mathrm{ha}^{-1}\right)$ and boll development stage $\left(0.09 \mathrm{~kg} \mathrm{ha}^{-1}\right)$, however, it was at 
par with $\mathrm{H}_{2} \mathrm{~F}_{7}, \mathrm{H}_{2} \mathrm{~F}_{9}, \mathrm{H}_{1} \mathrm{~F}_{7}, \mathrm{H}_{1} \mathrm{~F}_{8}$ and $\mathrm{H}_{1} \mathrm{~F}_{9}$ at square formation, $\mathrm{H}_{2} \mathrm{~F}_{7}, \mathrm{H}_{2} \mathrm{~F}_{9}, \mathrm{H}_{1} \mathrm{~F}_{8}$ and $\mathrm{H}_{1} \mathrm{~F}_{9}$ at boll formation and $\mathrm{H}_{2} \mathrm{~F}_{4}, \mathrm{H}_{2} \mathrm{~F}_{5}, \mathrm{H}_{2} \mathrm{~F}_{6}, \mathrm{H}_{2} \mathrm{~F}_{7}$ and $\mathrm{H}_{2} \mathrm{~F}_{9}$ at boll development stage.

DTPA iron: The iron content in the soil was decreased from square formation to boll development stages (Table 5). Non-Bt hybrid recorded maximum content in the soil (4.54 ppm), (4.40 ppm) and (3.40 $\mathrm{ppm})$ as compared to Bt cotton (4.50 ppm), (4.24 ppm) and $(3.18 \mathrm{ppm})$ at square formation, boll formation and boll development stage, respectively. The treatment $\mathrm{F}_{7}$ recorded maximum iron content in the soil (4.82 and $4.78 \mathrm{ppm}$, respectively) which was significantly superior over all the treatments tested at square and boll formation. At boll formation stage, $\mathrm{F}_{8}$ showed maximum DTPA extractable soil iron (3.56 ppm), which was significantly superior over all the tested treatments. As regards the interaction between $\mathrm{Bt} /$ non-Bt with different fertilizer doses was found to be significant at all growth stages. At square and boll formation stages, $\mathrm{H}_{2} \mathrm{~F}_{7}$ showed significantly higher DTPA extractable soil iron (4.84 and $4.80 \mathrm{ppm}$, respectively), however it was at par with $\mathrm{H}_{1} \mathrm{~F}_{7}$ (4.76 $\mathrm{ppm})$ at boll formation stage. However, at boll development $\mathrm{H}_{2} \mathrm{~F}_{8}$ showed significantly higher DTPA soil iron than other tested treatments. Mimmo et al.,(2014) reported that an enhanced release of inorganic (such as protons) and organic (organic acids, carbohydrates, amino acids, phytosiderophores, siderophores, phenolics and enzymes) compounds to increase the solubility of poorly available Fe pools.

Seed cotton yield: The Bt hybrid recorded maximum seed cotton yield $\left(29.07 \mathrm{q} \mathrm{ha}^{-1}\right)$ as compared to non-Bt hybrid (26.80 q ha ${ }^{-1}$ ) (Table 6). The treatment $\mathrm{F}_{8}\left(100 \% \mathrm{RDF}+1 \% \mathrm{MgSO}_{4}\right)$ had given higher seed cotton yield $\left(32.40 \mathrm{q} \mathrm{ha}^{-1}\right)$ which was at par with $\mathrm{F}_{9}$ $\left(100 \% \mathrm{RDF}+1 \% \mathrm{KNO}_{3}\right), \mathrm{F}_{7}(100 \% \mathrm{RDF}+2 \%$ DAP) and $\mathrm{F}_{6}(150 \% \mathrm{RDF})(31.17,30.42$ and $29.14 \mathrm{q}$ $\mathrm{ha}^{-1}$, respectively). The application of $100 \% \mathrm{RDF}$ along with foliar spray of $1 \% \mathrm{MgSO}_{4} / 1 \% \mathrm{KNO}_{3} / 2 \%$ DAP helped in reducing reddening of leaves in cotton, which was ultimately resulted in increasing the yield of cotton.The soil application of $\mathrm{MgSO}_{4} 25 \mathrm{~kg} \mathrm{ha}^{-1}+3$ foliar sprays of $1 \% \mathrm{MgSO}_{4}$ and 1\% 19:19:19 water soluble fertilizer along with RDF 100: $50: 50 \mathrm{~kg} \mathrm{NPK}$ $\mathrm{ha}^{-1}+$ FYM $10 \mathrm{t} \mathrm{ha}^{-1}$ recorded significantly higher seed cotton yield (2781 $\mathrm{kg} \mathrm{ha}^{-1}$ ) as compared to other nutritional treatments and it was on par with the soil application of $\mathrm{MgSO}_{4} 25 \mathrm{~kg} \mathrm{ha}^{-1}+3$ foliar sprays of $1 \% \mathrm{MgSO}_{4}$ and $2 \% \mathrm{KNO}_{3}$ water soluble fertilizer along with RDF +FYM (Shivamurthy and Biradar, 2014). Nehra et al., (2004) indicated that the application of $50 \% \mathrm{NPK}+10 \mathrm{t}$ FYM ha ${ }^{-1}+$ foliar nutrition was recorded the highest seed cotton yield (1940 $\mathrm{kg} \mathrm{ha}^{-1}$ ) and remained statistically on par with $100 \%$ NPK+5 t FYM ha ${ }^{-1}, 50 \%$ NPK+10 t FYM ha ${ }^{-1}$ and $100 \%$ NPK+10 t FYM ha ${ }^{-1}$. Singh et al. (2004) reported that the highest seed cotton yield was obtained with $\mathrm{KNO}_{3}$, followed by $2 \%$ urea and these two treatments were at par with each other. Similarly, Prabhakar (1981) reported that leaf reddening has been found to be a serious physiological problem in cotton resulting in reduced yield to an extent of 50 per cent depending on the extent of incidence. The interaction between $\mathrm{Bt} /$ non-Bt with different fertilizer doses was found non-significant. On the basis of this trial it was concluded that the application of two foliar spray of $1 \% \mathrm{MgSO}_{4}$ at boll development and 15 days thereafter first spray along with $100 \% \mathrm{RDF}+\mathrm{FYM}$ or two foliar spray of $1 \%$ $\mathrm{KNO}_{3}$ at boll development and 15 days thereafter first spray along with $100 \% \mathrm{RDF}+\mathrm{FYM}$ or two foliar spray of $2 \%$ DAP at boll development and 15 days thereafter first spray along with $100 \%$ RDF + FYM appears to be better for increasing nutrient availability and yield of Bt cotton.

\section{REFERENCES}

Anonmyous (2013).The Cotton Corporation of India Ltd. $43^{\text {rd }}$ Annual Report 2012-2013. pp-7.

Anonmyous (2014). Cotton Industry India, Ministry of Textiles, Government of India.

Islamov, I.I. and Ishmakhamedova, S.G. (1967). Application of potassium to cotton. Khlop Kovodstvo. $10: 30$ (Quoted from F.C.A. 1968 No. 1128).

Jadhao, J.G., Jadhao, S.D., Ghodpage, R.M. and Ingole, A.S. (2004). Effect of different chemical sprays on reddening and morphological characters in cotton. PKV Research Journal, 28(2):225-228.

Jayakumar, M. Surendran, U. and Manickasundaram, P. (2014). Drip fertigation effects on yield, nutrient uptake and soil fertility of Bt Cotton in semi arid tropics. International Journal of Plant Production, 8 (3):375 -390.

Mimmo, T., Del Buono, D., Terzano, R., Tomasi, N., Vigani, G., Crecchio, C., Pinton, R., Zocchi, G. and Cesco, S. (2014). Rhizospheric organic compounds in the soil microorganism plant system: their role in iron availability. European Journal of Soil Science. doi: 10.1111/ejss.12158.

Nehra, P. L. Kumawat P. D. and Nehara, K. C. (2004). Integrated nutrient management in hirsutum cotton. Journal of Cotton Research Development, 18(2): 177179.

Panse, V.G. and Sukhatme, P.V. (1985). Statistical methods for agricultural workers, ICAR Publication, New Delhi.

Prabhakar, A. S. (1981). Agronomic investigation on irrigated cotton Ph.D. Thesis, University of Agricultural Sciences, Banglore.

Ravankar, H. N., Sujata, P. A. and Hadole, S. S. (2001). Distribution of organic matter in vertisols under long term fertilization to sorghum-wheat cropping sequence. PKV Research Journal, 25(1): 16-19.

Sekhon, N. K and Singh, C. B. (2013). Plant nutrient status during boll development and seed cotton yield as affected by foliar application of different sources of potassium. American Journal of Plant Sciences, 4:1409-1417.

Shivamurthy, D. and Biradar, D. P. (2014). Effect of foliar nutrition on growth, yield attributes and seed cotton yield of Bt cotton. Karnataka Journal of Agricultural 
Sciences, 27 (1): 5-8.

Singh, A., Rathore, P. and Pathak, D. (2004). Effect of foliar application of inorganic nutrients on yield of American cotton. Journal of Cotton Research Development, 18 (2): 169-171.

Singh, J. and Kaushik, S. K. (2007). Bt cotton in India. Present - scenario and future prospects. Indian Farming, 56(11): 26-28.
Tewolde, H., Adeli, A. Sistani, K.R. and Rowe, D. E. (2010). Potassium and magnesium nutrition of cotton fertilized with broiler litter. The Journal of Cotton Science, 14:1 -12 .

Tinker, P. B. (1980). Role of rhizosphere microorganisms in phosphorus uptake by plants, In: R.C. Dinauer (Ed.), The Role of Phosphorus in Agriculture, ASA-CSSASSSA, Madison, WI, USA. 